\title{
25 Research Soure \\ Anesthesia and Intubation of 10-Day Old C57BL/6J Mouse Pups for Cardiothoracic Surgery
}

\author{
Jianxin Wu \\ Victor Chang Cardiac Research Institute \\ Amy M. Nicks \\ Victor Chang Cardiac Research Institute \\ Justin J. Skowno \\ Children's Hospital at Westmead \\ Michael P. Feneley \\ Victor Chang Cardiac Research Institute \\ Siiri E. lismaa ( $\nabla$ s.iismaa@victorchang.edu.au ) \\ Victor Chang Cardiac Research Institute \\ Robert M. Graham \\ Victor Chang Cardiac Research Institute
}

\section{Research Article}

Keywords: mouse pup, anesthesia, intubation, myocardial infarction, cannibalism, oxygenation

Posted Date: December 15th, 2021

DOI: https://doi.org/10.21203/rs.3.rs-1144085/v1

License: (c) (i) This work is licensed under a Creative Commons Attribution 4.0 International License.

Read Full License 


\section{Abstract}

Murine surgical models play an important role in preclinical research. Mechanistic insights into myocardial regeneration after cardiac injury may be gained from cardiothoracic surgery models in 0-14day-old mice, the cardiomyocytes of which, unlike those of adults, retain proliferative capacity. Mouse pups up to 7 days old are effectively immobilized by hypothermia and do not require intubation for cardiothoracic surgery. Preadolescent (8-14-day-old) mouse pups, however, do require intubation, but this is challenging and there is little information regarding anesthesia to facilitate intubation. Empirical titration of ketamine/xylazine/atropine dosage regimens to body weight indicated the response to anesthesia of 10-day-old C57BL6/J mouse pups of different weights was non-linear, whereby doses of $20 / 4 / 0.12 \mathrm{mg} / \mathrm{kg}, 30 / 4 / 0.12 \mathrm{mg} / \mathrm{kg}$ and $50 / 6 / 0.18 \mathrm{mg} / \mathrm{kg}$, facilitated intubation of pups weighing between 3.15-4.49 $\mathrm{g}(\mathrm{n}=22), 4.50-5.49 \mathrm{~g}(\mathrm{n}=20)$ and 5.50-8.10 $\mathrm{g}(\mathrm{n}=20)$, respectively. Lower-body-weight pups required more intubation attempts than heavier pups $(\mathrm{p}<0.001)$. Survival post-intubation was inversely correlated with body weight $(65,70$ and $80 \%$ for low-, mid- and high-weight groups, respectively, $\left.\mathrm{R}^{2}=0.995\right)$. For myocardial infarction surgery after intubation, a surgical plane of anesthesia was induced with $4.5 \%$ isoflurane in $100 \%$ oxygen and maintained with $2 \%$ isoflurane in $100 \%$ oxygen. Survival postsurgery was similar for the three weight groups at $92 \%, 86 \%$ and $88 \%(p=0.91)$. Together with refinements in animal handling practices for intubation and surgery, and to minimize cannibalization by the dam postsurgery, overall survival for the entire procedure (intubation plus surgery) was inversely correlated with body weight ( $55 \%, 60 \%$ and $70 \%$ for low-, mid- and high-weight groups, respectively, $\left.\mathrm{R}^{2}=0.978\right)$. Given the difficulty encountered with intubation of 10-day old pups and the associated high mortality, we recommend cardiothoracic surgery in 10-day-old pups be restricted to those weighing at least $5.5 \mathrm{~g}$.

\section{Introduction}

Murine models are invaluable tools in preclinical research, in particular because of the ease with which genetically-engineered mouse lines can be generated or mice can be surgically manipulated to provide pathological disease models to allow, for example, the study of myocardial regeneration after cardiac injury. ${ }^{1}$ In this regard, it is of interest that unlike adult mice, in which cardiomyocytes have withdrawn from the cell cycle, 0-2-day-old neonatal mouse hearts repair with minimal scarring after apical resection or induction of myocardial infarction. ${ }^{2-4}$ In contrast, 7-day-old neonatal hearts regenerate incompletely with a higher incidence of scarring. ${ }^{2,3}$ Since cardiomyocytes in the apex of the left ventricle retain proliferative capacity for up to two weeks after birth, mechanistic studies of regeneration after cardiac injury in 0-14-day-old mice may be informative for identifying therapeutic targets for regeneration of the injured adult heart. ${ }^{5}$

The development of mouse models of cardiac injury involves surgical manipulation under anesthesia. This requires that the thorax be opened to access the heart, which generally mandates intubation and mechanical ventilation. Mouse strain, body weight and age influence sensitivity to anesthetics. ${ }^{6}$ Adult mice can be anesthetised with a wide range of agents, a common regimen for intubation being 
ketamine/xylazine/atropine (100/13/0.5 mg/kg, respectively) ${ }^{6,7}$ Neonatal mice (0-7 days old) lack centralised pain reflexes, and can be effectively immobilised on ice and subjected to surgery without intubation. ${ }^{6,8,9}$ Preadolescent (8-14-day-old) mouse pups cannot be anesthetised with hypothermia and require intubation for cardiothoracic surgery, but intubation is difficult and there is little information on anesthetic regimens that both facilitate intubation and maximise survival for animals of this age. ${ }^{9,10}$ Mice of this age are still developing neurologically and their responses to drugs and drug metabolism are very different from adult animals. ${ }^{6}$ This poses increased risk of fluid, electrolyte and acid-base imbalance, as well as hypoglycemia and hypothermia due not only to their high metabolic rate, which rapidly depletes their limited energy stores, but also to their thermoregulatory immaturity. $6,11,12$

Here we empirically titrated dosage regimens of ketamine/xylazine/atropine in 10-day-old C57BL/6J mouse pups ranging in weight from 3-8 $\mathrm{g}$ to achieve a plane of anesthesia sufficient to allow endotracheal intubation for subsequent cardiothoracic surgery, while minimising animal mortality. We also refined animal handling practices to reduce mortality from intubation, surgery and post-surgical maternal cannibalism.

\section{Results}

Anesthesia of 10-day old mice. Ten-day-old pups can be anesthetised with $4.5 \%$ isoflurane in $4-5$ mins, however, due to their small size, intubation under isoflurane anesthesia, delivered by a standard nose cone, is not feasible. We have previously used a ketamine/xylazine/atropine anesthetic regimen of $100 / 13 / 0.5 \mathrm{mg} / \mathrm{kg}$, respectively, for thoracic surgery in 15- and 21-day-old pups and adults. ${ }^{4,7}$ In preliminary experiments that included oxygen supplementation we found this regimen resulted in an unacceptable mortality in 10-day-old pups. Given the inverse correlation between body weight and litter size of 10 -day-old pups $\left(R^{2}=0.250, p<0.0001\right.$; Figure 1$)$, we titrated the anesthetic regimen according to body weight groupings. Reducing the ketamine/xylazine/atropine dosage to 50/6/0.18 mg/kg, respectively, resulted in a sufficient depth of anesthesia to allow endotracheal intubation of spontaneously breathing pups weighing $5.5-8.10 \mathrm{~g}$ (Table 1 ) but this dose was not tolerated by lighter pups. Reducing the ketamine/xylazine/atropine dosage to $30 / 4 / 0.12 \mathrm{mg} / \mathrm{kg}$ enabled intubation of pups weighing 4.50-5.49 g, while further reduction of the ketamine dosage to $20 \mathrm{mg} / \mathrm{kg}$ enabled intubation of pups weighing $3.15-4.49 \mathrm{~g}$ (Table 1 ). 
Table 1

Anesthesia regimen, number of intubation attempts and post-procedure survival of 10-day-old mouse pups

\begin{tabular}{|c|c|c|c|c|c|c|c|c|}
\hline \multirow{2}{*}{$\begin{array}{l}\text { Anesthesia } \\
\text { regimen: } \\
\text { ketamine/ } \\
\text { xylazine/ } \\
\text { atropine }(\mathrm{mg} / \mathrm{kg}) \text {; } \\
\text { given in } 10 \mathrm{~mL} / \mathrm{g} \\
\text { body weight, ip }\end{array}$} & \multirow[t]{2}{*}{$\begin{array}{l}\text { Body } \\
\text { weight, } \\
\text { g }\end{array}$} & \multirow[t]{2}{*}{$\begin{array}{l}\text { Number } \\
\text { of pups } \\
\text { studied }\end{array}$} & \multicolumn{3}{|c|}{$\begin{array}{l}\text { Intubation } \\
\text { attempts (A, 1-2; B, } \\
3-4 \text { or C, >4) and } \\
\text { number of pups } \\
\text { intubated, } n(\%)\end{array}$} & \multirow[t]{2}{*}{$\begin{array}{l}\text { Intubated } \\
\text { pups } \\
\text { proceeding } \\
\text { to surgery, } \\
\text { n (\%) }\end{array}$} & \multirow[t]{2}{*}{$\begin{array}{l}\text { Survival } \\
\text { two } \\
\text { days } \\
\text { post- } \\
\text { surgery, } \\
\text { n (\%) }\end{array}$} & \multirow{2}{*}{$\begin{array}{l}\text { Overall } \\
\text { survival } \\
\text { after } \\
\text { intubatior } \\
\text { plus } \\
\text { surgery, } n \\
(\%)\end{array}$} \\
\hline & & & A & B & $\mathrm{C}$ & & & \\
\hline $20 / 4 / 0.12$ & $\begin{array}{l}3.15- \\
4.49\end{array}$ & 22 & $\begin{array}{l}8 \\
(36)\end{array}$ & $\begin{array}{l}9 \\
(41)\end{array}$ & $\begin{array}{l}5 \\
(23)\end{array}$ & $13(59)$ & $12(92)$ & $12(55)$ \\
\hline $30 / 4 / 0.12$ & $\begin{array}{l}4.50- \\
5.49\end{array}$ & 20 & $\begin{array}{l}13 \\
(65)\end{array}$ & $\begin{array}{l}5 \\
(25)\end{array}$ & $\begin{array}{l}2 \\
(10)\end{array}$ & $14(70)$ & $12(86)$ & $12(60)$ \\
\hline $50 / 6 / 0.18$ & $\begin{array}{l}5.50- \\
7.30\end{array}$ & 20 & $\begin{array}{l}13 \\
(65)\end{array}$ & $\begin{array}{l}3 \\
(15)\end{array}$ & $\begin{array}{l}4 \\
(20)\end{array}$ & $16(80)$ & $14(88)$ & $14(70)$ \\
\hline \multicolumn{3}{|l|}{$\mathrm{p}$ (Chi-square test) } & \multicolumn{3}{|c|}{$p<0.001$} & & \multicolumn{2}{|l|}{$p=0.91$} \\
\hline \multirow{2}{*}{\multicolumn{6}{|c|}{$\begin{array}{l}R^{2} \text { (Correlation coefficient, } \\
p \text { value) }\end{array}$}} & 0.995, & & 0.978 \\
\hline & & & & & & $p=0.04$ & & $p=0.09$ \\
\hline
\end{tabular}

Intubation of 10-day old mice. Before intubation, the depth of anesthesia was assessed by the paw pinch reflex. For optimal intubation, the paw pinch reflex had to still be present but markedly reduced from that of a conscious animal. Intubation of 10-day old pups required specialised equipment (Figure 2A-C). After securing the anesthetised pup supine to an intubation platform (Figure 2B), the tongue was held with small forceps and a laryngoscope fashioned out of a piece of copper wire (Figure 2C) was used to expose the glottis and vocal cords, visualisation of the vocal cords being aided by trans-illumination with a flexible fibre-optic light (Figure 2D). A 24-gauge plastic intravenous (iv) cannula was used as an endotracheal tube. This consisted of a $19 \mathrm{~mm}$ length of plastic tubing ( $0.7 \mathrm{~mm}$ OD) attached to a $21 \mathrm{~mm}$ plastic female luer lock adaptor (Figure 2B). The total volume of the cannula was $130 \mathrm{~mL}$; the tidal volume of a mouse being $\sim 8 \mathrm{~mL} / \mathrm{g} .{ }^{13,14}$ At the time of intubation, the tubing of the cannula was stiffened by insertion of a piece of copper wire via the luer lock adaptor (Figure 2B); the wire being removed immediately after intubation. The cannula, tilted so that the luer lock end was slightly lower $\left(\sim 10^{\circ}\right)$ than the tip, was inserted as soon as the vocal cords separated.

The depth of anesthesia was assessed after intubation by the ability of the animal to breathe spontaneously. Successful tracheal intubation of spontaneously breathing pups was confirmed by briefly blocking the intubation catheter to check that this prevented chest movement. The endotracheal cannula was then connected to a ventilator delivering $100 \%$ oxygen. These procedures were performed rapidly ( $<15 \mathrm{sec}$ ) to minimise re-breathing. 
Outcomes were best when intubation was achieved after only one or two attempts. Pups with a lower body weight were more difficult to intubate than heavier pups and required more attempts $(p<0.001$; Table 1). Survival post-intubation was inversely correlated with body weight $(65,70$ and $80 \%$ for low-, mid- and high-weight groups, respectively, $R^{2}=0.995, p=0.04$; Table 1 ).

Myocardial infarction surgery of 10-day old mice. For myocardial infarction surgery after intubation, anesthesia was induced with $4.5 \%$ isoflurane in $100 \%$ oxygen and tracheal intubation again confirmed by checking that the frequency of chest wall movement equalled that of the ventilator. Loss of spontaneous breathing followed by absence of a tail or paw pinch reflex indicated that a surgical plane of anesthesia had been reached (after 4-5 min). Anesthesia was maintained with $2 \%$ isoflurane. The thorax was opened, and a myocardial infarction was induced by ligation of the left anterior descending coronary artery just distal to the left atrial appendage. After the $~ 10$ min surgery, animals were allowed to recover by discontinuing the isoflurane. Spontaneous breathing resumed within a few minutes thereafter and then the pup was returned to the warmed pre-oxygenated chamber and monitored continuously during recovery until the righting reflex was regained, at which point the pup was extubated.

Of the pups that did not survive to follow-up at $48 \mathrm{~h}$ (Table 1), one from the low-weight group died $6 \mathrm{~h}$ after surgery, one pup from each of the mid- and high-weight groups died before being placed back with the dam, and one pup from each of the mid- and high-weight groups was cannibalized by the dam within $16 \mathrm{~h}$ of surgery, with small body parts or nothing remaining the next morning. Post-surgery cannibalism by the dam was reduced if pups were cleaned of blood and disinfectant, gently rubbed with home cage bedding, kept warm, were breathing regularly and were capable of spontaneous movement i.e. had fully recovered from anesthesia, before returning the dam to the cage.

Survival two days after myocardial infarction surgery was consistent between the different weight groups at $86-92 \%$ ( $p=0.91$; Table 1$)$. Infarcted myocardium, as assessed two days post-surgery by Alcian-blue perfusion of the heart, was evident by clear demarcation of stained, non-infarcted (blue) from ischaemic (unstained) tissue, distal to the ligation (Figure 2E).

Overall survival for the entire procedure (intubation plus surgery) was inversely correlated with pup body weight ( $55 \%, 60 \%$ and $70 \%$ for low-, mid- and high-weight groups, respectively, $\left(R^{2}=0.978\right.$, Table 1$)$, although this correlation did not achieve statistical significance $(p=0.09)$.

\section{Discussion}

Currently, there are no well-documented methods for anesthesia and intubation of 10-day old mice for cardiothoracic surgery. To this end, we have titrated ketamine/xylazine/atropine dosage regimens to body weight, whereby doses of 20/4/0.12 mg/kg, 30/4/0.12 mg/ $\mathrm{kg}$ and $50 / 6 / 0.18 \mathrm{mg} / \mathrm{kg}$ facilitated intubation of pups with low (3.15-4.49), mid (4.50-5.49) and high (5.50-8.10 g) body weight, respectively. Survival post-intubation was inversely correlated with body weight $(65,70$ and $80 \%$ for low-, mid- and high-weight groups, respectively. Given the difficulty encountered with intubation of 10-day old pups and 
the associated high mortality, we recommend cardiothoracic surgery in 10-day-old pups be restricted to animals weighing at least $5.50 \mathrm{~g}$. Overall pup survival after intubation and myocardial infarction surgery ranged from 55\% (lowest body weight group) to 70\% (highest body weight group. This is similar to the 60$70 \%$ survival reported for 1 -day-old pups, which require no intubation when subjected to myocardial infarction after immobilization on ice. ${ }^{8}$

We found 10-day-old mouse pups of different weights had a non-linear response to the ketamine/xylazine/atropine anesthetic regimen. This may reflect developmental differences in a number of important areas. Basal metabolic rate scales allometrically to the three-quarter power of mass, from single cells to mammals. ${ }^{15}$ This would influence drug disposition in the animals in our study, which varied in weight by 2.5 times. The maturity of drug metabolism or detoxification mechanisms is another factor that changes rapidly in the immediate postnatal period, as are mechanisms influencing free drug availability, such as protein binding. ${ }^{16}$ Pharmacokinetic differences may not be the only explanation for non-linear drug-effect relationships, as differences in pharmacodynamic responses to sedative agents are also possible. ${ }^{6}$ The use of oxygenation after intraperitoneal injection of anesthetics and prior to intubation likely improved the safety of the procedure, as has been noted recently for adults. ${ }^{17}$ Further adjustments in dosage, particularly for the lowest body weight group, may improve survival.

The depth of anesthesia was critical for successful intubation. Intubation was difficult if the plane of anesthesia was too light, and if too deep, pups stopped breathing spontaneously, either during intubation or after intubation while being ventilated with oxygen. Handling of pups also sometimes caused breathholding, especially during intubation. If breathing stopped during intubation, stimulation of the foot or tail, or returning pups to the warmed oxygen-filled chamber was critical to restore regular breathing. Intubation was re-attempted when the pup resumed regular breathing. If breathing stopped after intubation, the animals were ventilated for up to $10 \mathrm{~min}$ with oxygen. If spontaneous breathing resumed during this time, the animals proceeded to surgery. However, we found that if spontaneous breathing was not restored within this time, pups did not recover from anesthesia or, if subjected to surgery, died during the recovery period.

Given the high metabolic rate of 10-day-old pups, it is best to limit depletion of energy stores by separating the dam from its pups for as short a time as possible and, thus, restricting the number of surgical operations to 4-5 pups per litter per day. Any littermates that did not undergo surgery were removed to foster mothers or culled before return of the dam to the cage. Our handling practices to reduce mortality from post-surgical maternal cannibalism were similar to those that have been reported for neonates. ${ }^{9}$

\section{Conclusions}

Our feasibility study suggests that an injectable ketamine/xylazine/atropine anesthetic regimen considerably lower than that used for older mice is required to minimize mortality from intubation of 10- 
day-old mouse pups for subsequent cardiothoracic surgery, as are specific handling practices to reduce mortality from intubation, surgery, and post-surgical maternal cannibalism.

\section{Materials And Methods}

Ethics statement. C57BL/6J mice were maintained under a 12-hour light/dark cycle with ad libitum food and water. Procedures were approved by the Garvan/St Vincent's Hospital Animal Ethics Committee (AEC19/17) in accordance with the Australian Code of Practice for the Care and Use of Animals for Scientific Purposes and the ARRIVE guidleines, and all experiments were performed by an experienced small animal surgeon (JW) with guidance from a pediatric anesthetist (JJS).

Anesthesia. On the day of surgery, the dam was removed from a cage of 10-day-old pups and the cage was placed on a warming pad $\left(37^{\circ} \mathrm{C}\right)$. Pups were anesthetised by intraperitoneal injection $(10 \mathrm{~mL}$ per $\mathrm{g}$ body weight, $0.5 \mathrm{~mL}$ insulin syringe and $29 \mathrm{G}$ needle (BD Ultra-Fine U-100)) with ketamine/xylazine/atropine in the ratios detailed in Table 1 for different weight groupings. Immediately after injection, the pup was placed into a warmed $\left(37^{\circ} \mathrm{C}\right)$ plexiglass chamber that had been preoxygenated with $100 \%$ oxygen.

Intubation. After 3-4 min of oxygenation, the pup was transferred to a platform for intubation in the supine position essentially as described for adult mice. ${ }^{18}$ Temperature was maintained with a warming light. Intubated pups were immediately transferred to a warming pad $\left(37^{\circ} \mathrm{C}\right)$ and the endotracheal cannula connected to a ventilator delivering $100 \%$ oxygen at a flow rate of $1 \mathrm{~L} / \mathrm{min}(30,40$ or 50 $\mathrm{mL} /$ stroke for $3.15-4.59 \mathrm{~g}, 4.50-5.49 \mathrm{~g}$, or 5.50-8.10 g pups, respectively; 150 strokes/min).

Surgery. To induce a surgical plane of anesthesia for surgery, the gas flowing into the ventilator was switched from $100 \%$ oxygen to $4.5 \%$ isoflurane in oxygen (the isoflurane concentration being determined by a vaporiser) for $4-5 \mathrm{~min}$. Anesthesia was then maintained with $2 \%$ isoflurane in $100 \%$ oxygen. Myocardial infarction surgery was performed under a surgical microscope (Leica M651, 10x and 16x objective) essentially as described. ${ }^{19}$ The left anterior descending coronary artery was ligated with 9-0 polypropylene monofilament suture and the skin closed with 7-0 suture. Analgesia (buprenorphine, $0.075 \mathrm{mg} / \mathrm{kg}, \mathrm{sc}$ ) and saline (50 mL, ip) were administered after surgery. The animal was extubated when the righting reflex was regained. Animals were housed overnight in a cage placed half on/half off a $37^{\circ} \mathrm{C}$ warming pad. Pups were monitored for two days after surgery. There were no signs of pain post-surgery.

Post-surgery assessment of infarct size. Two days after surgery, pups were sacrificed, and the hearts harvested to visualise infarcts. To this end, pups were anesthetised by placing them in a plexiglass chamber pre-equilibrated with $4.5 \%$ isoflurane in oxygen $(1 \mathrm{~mL} / \mathrm{min}$ flow rate). Once a surgical plane of anesthesia had been reached (after 4-5 $\mathrm{min}$ ), the pup was removed from the chamber and secured supine onto a warming pad by taping the tail. A thread was placed over the incisors and taped into position to keep the head extended, and the head was placed into a nose cone connected to a ventilator delivering $4.5 \%$ isoflurane in oxygen $(200 \mathrm{~mL} / \mathrm{stroke}, 150$ strokes/min). A surgical plane of anesthesia 
was maintained with $2 \%$ isoflurane in oxygen. An incision was made in the skin over the right common carotid artery along the trachea and the exposed vessel cannulated (single lumen polyethylene tube; OD $0.61 \mathrm{~mm}$, ID $0.28 \mathrm{~mm}$; Critchley Electrical Products Pty Ltd Auburn NSW) for administration of heparinized saline $(0.2 \mathrm{~mL}, 200 \mathrm{U}, 1 \mathrm{~min})$ to prevent blood clotting. Isoflurane was increased to $4.5 \%$ in oxygen for $1 \mathrm{~min}$ before rapid administration of $\mathrm{KCl}(0.2 \mathrm{~mL}, 3.3 \mathrm{M}, 2 \mathrm{sec})$ to arrest the heart in diastole. The right jugular vein was cut to allow blood to be washed out by perfusion of the heart with phosphatebuffered saline (PBS, $0.2 \mathrm{~mL})$, and then Alcian Blue $(0.1 \mathrm{~mL}, 0.2 \%)$ was perfused to stain the non-infarcted remote myocardium. The heart was excised and rinsed with PBS, the atria were removed, and the heart was photographed with a camera (Leica, IC80 HD) mounted on a surgical microscope (Leica, M651, $10 \mathrm{x}$ objective).

Statistics. Power calculations to prespecify sample sizes were not possible because this was an exploratory study. There were no exclusion criteria, and no data were excluded. Data were analysed by Chi-squared test and simple linear regression, with $p<0.05$ being considered significant (GraphPad Prism, Version 9.1.2, GraphPad Software, LLC).

\section{List Of Abbreviations}

iv, intravenous

ip, intraperitoneal

PBS, phosphate-buffered saline

\section{Declarations}

\section{Availability of data and materials}

The datasets supporting the conclusions of this article are included within the article.

\section{Acknowledgements}

This work was supported by NHMRC Program Grant [ID 1074386], a Leducq Transatlantic Network of Excellence in Cardiovascular Research grant [RMG] and a grant from the RT Hall Trust [RMG \& SEI].

\section{Author's contributions}

RMG conceived the study; JW performed the study with input from SEI, AMN, JJS, MPF and RMG; JW and SEl analysed and interpreted the data, and wrote the manuscript; all authors edited the manuscript and approved the submitted version.

\section{Competing interests}

The authors declare that they have no competing interests 


\section{References}

1. Klocke, R., Tian, W., Kuhlmann, M. T. \& Nikol, S. Surgical animal models of heart failure related to coronary heart disease. Cardiovasc Res 74, 29-38, doi:10.1016/j.cardiores.2006.11.026 (2007).

2. Porrello, E. R. et al. Transient regenerative potential of the neonatal mouse heart. Science 331, 10781080, doi:10.1126/science.1200708 (2011).

3. Porrello, E. R. et al. Regulation of neonatal and adult mammalian heart regeneration by the miR-15 family. Proc Natl Acad Sci U S A 110, 187-192, doi:10.1073/pnas.1208863110 (2013).

4. Naqvi, N. et al. A proliferative burst during preadolescence establishes the final cardiomyocyte number. Cell 157, 795-807, doi:10.1016/j.cell.2014.03.035 (2014).

5. Bogush, N. et al. DUSP5 expression in left ventricular cardiomyocytes of young hearts regulates thyroid hormone (T3)-induced proliferative ERK1/2 signaling. Sci Rep 10, 21918, doi:10.1038/s41598-020-78825-x (2020).

6. Flecknell, P. Laboratory animal anaesthesia. Third edn, (Elsevier Inc, 2009).

7. lismaa, S. E. et al. Cardiac hypertrophy limits infarct expansion after myocardial infarction in mice. Sci Rep 8, 6114, doi:10.1038/s41598-018-24525-6 (2018).

8. Mahmoud, A. I., Porrello, E. R., Kimura, W., Olson, E. N. \& Sadek, H. A. Surgical models for cardiac regeneration in neonatal mice. Nat Protoc 9, 305-311, doi:10.1038/nprot.2014.021 (2014).

9. Boston University Institutional Animal Care and Use Committee. Anesthesia and Analgesia: Neonatal Mice and Rats, <https://www.bu.edu/researchsupport/compliance/animal-care/working-withanimals/anesthesia/anesthesia-and-analgesia-neonatal-mice-and-rats-iacuc/> (2019).

10. University of Texas at Austin Animal Resources Center. Mouse-Specific Anesthesia Guidance, <https://research.utexas.edu/wpcontent/uploads/sites/7/2020/02/Mouse_Anesthesia_guidance_ARC_112519.pdf> (2019).

11. Paddleford, R. Manual of small animal anaesthesia. Second edition edn, (Masson, 2000).

12. Gargiulo, S. et al. Mice anesthesia, analgesia, and care, Part I: anesthetic considerations in preclinical research. ILAR J 53, E55-69, doi:10.1093/ilar.53.1.55 (2012).

13. Daubenspeck, J. A., Li, A. \& Nattie, E. E. Acoustic plethysmography measures breathing in unrestrained neonatal mice. J Appl Physiol (1985) 104, 262-268, doi:10.1152/japplphysiol.00893.2007 (2008).

14. Lim, R. et al. Measuring respiratory function in mice using unrestrained whole-body plethysmography. J Vis Exp, e51755, doi:10.3791/51755 (2014).

15. West, G. B., Woodruff, W. H. \& Brown, J. H. Allometric scaling of metabolic rate from molecules and mitochondria to cells and mammals. Proc Natl Acad Sci U S A 99 Suppl 1, 2473-2478, doi:10.1073/pnas.012579799 (2002).

16. Moscovitz, J. E. \& Aleksunes, L. M. Establishment of metabolism and transport pathways in the rodent and human fetal liver. Int J Mol Sci 14, 23801-23827, doi:10.3390/ijms141223801 (2013). 
17. Blevins, C. E., Celeste, N. A. \& Marx, J. O. Effects of Oxygen Supplementation on Injectable and Inhalant Anesthesia in C57BL/6 Mice. J Am Assoc Lab Anim Sci 60, 289-297, doi:10.30802/AALASJAALAS-20-000143 (2021).

18. Brown, R. H., Walters, D. M., Greenberg, R. S. \& Mitzner, W. A method of endotracheal intubation and pulmonary functional assessment for repeated studies in mice. J Appl Physiol (1985) 87, 2362-2365, doi:10.1152/jappl.1999.87.6.2362 (1999).

19. Du, X.-J., Gao, X. \& Ramsey, D. Surgical methods of inducing transverse aortic stenosis and myocardial infarction in the mouse. Asia Pacific Heart J 7, 187-192 (1998).

20. Silver, L. M. Mouse Genetics: Concepts and Applications. (Mouse Genome Informatics, The Jackson Laboratory, 2008).

\section{Figures}

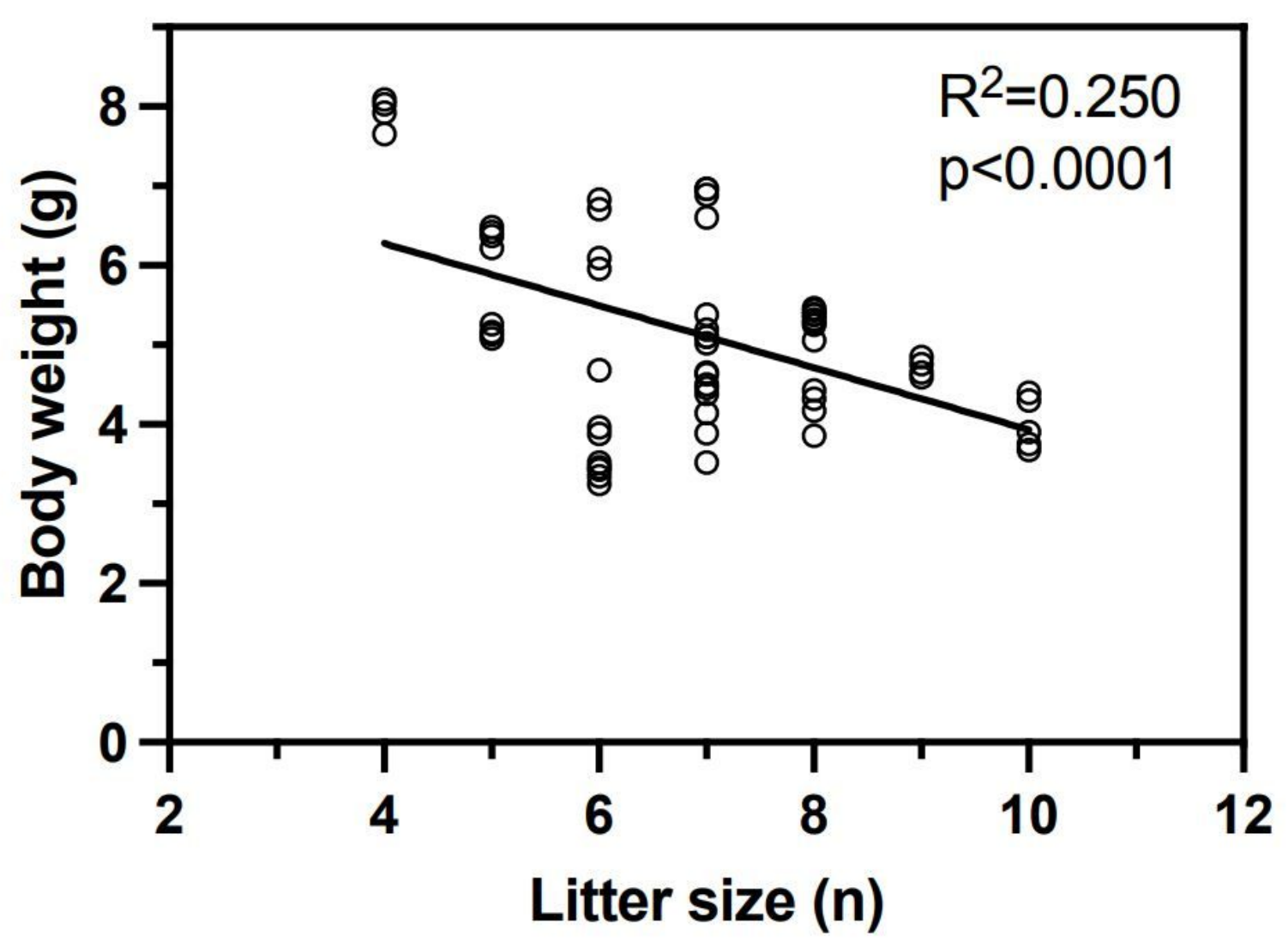

\section{Figure 1}

Body weight of 10-day-old C57BL/6 pups is inversely correlated with litter size. Pups were from litters ranging in size from $4-10$, the average litter size being 7.20 

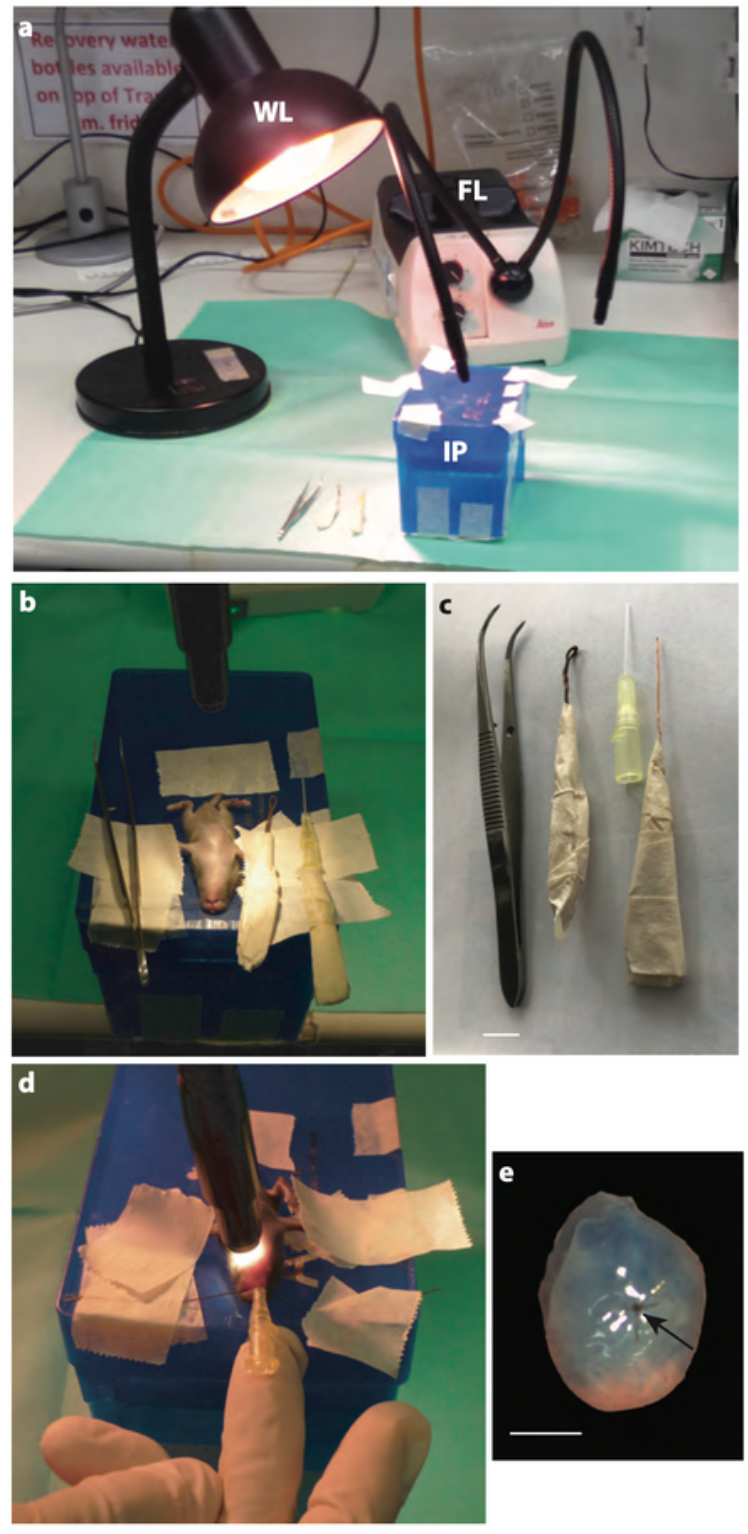

\section{Figure 2}

Endotracheal intubation of a 10-day-old C57BL/6J mouse pup. a Intubation set-up showing large warming light (WL), intubation platform (IP) and flexible fibre-optic lighting (FL; Leica CLS 150X) used to aid visualization of the vocal cords at the time of intubation). $b$ The anesthetised pup is secured supine by taping the tail and front limbs onto the intubation platform $(8.5 \times 12 \times 7.5 \mathrm{~mm})$. A thread placed over the incisors is used to extend the head and is taped in position. c forceps, laryngoscope, 24-gauge 
cannula (Multigate Medical Devices, Polywin IV cannula) that is used as an endotracheal tube, and a piece of copper wire that is inserted into the endotracheal tube via the luer lock adaptor to stiffen the cannula during intubation (scale bar $1 \mathrm{~cm}$ ). $d$ The fibre-optic light is placed over the neck to transilluminate the trachea just below the vocal cords. The tongue is held with small forceps, and then movement of the vocal cords visualised by exposing the glottis with the laryngoscope. The endotracheal tube is inserted into the trachea while the vocal cords are open. e Photograph of a representative Alcianblue perfused mouse pup heart (frontal view with the base of the heart at the top and apex at the bottom, and atria removed) $48 \mathrm{~h}$ post-ligation (black suture, black arrow) of the left anterior descending artery taken under a surgical microscope (Leica, M651, $10 \times$ objective) mounted with a camera (Leica, IC80 HD). Non-infarcted myocardium is stained blue, infarcted myocardium at the apex is unstained and pale; scale bar, $100 \mu \mathrm{m}$. 